
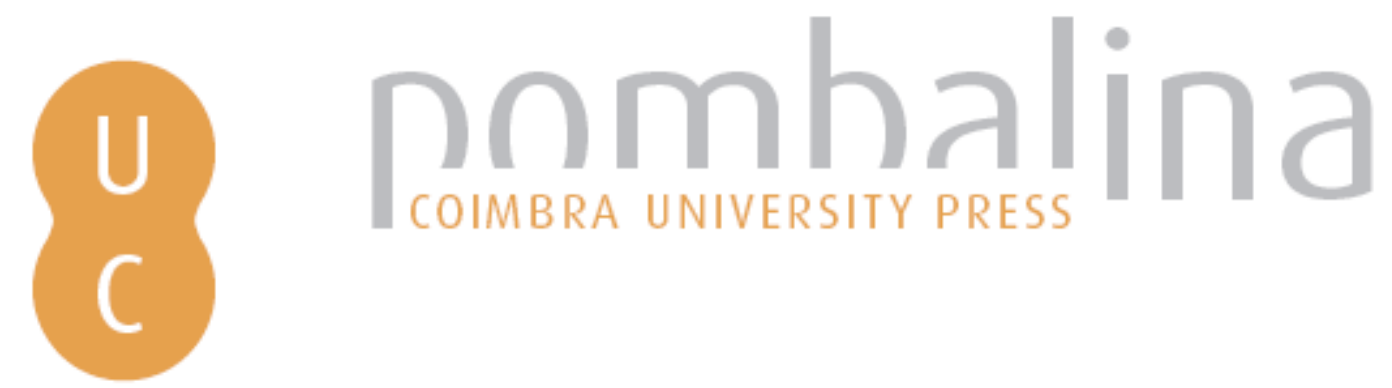

\title{
Otimização da operação de redes de distribuição: consideração simultânea de variáveis topológicas, tomadas de transformadores e baterias de condensadores utilizando EPSO e heurísticas
}

Autor(es): $\quad$ Pereira, Jorge; Alves, Jorge; Matos, Manuel

Publicado por: Imprensa da Universidade de Coimbra

URL persistente:

URI:http://hdl.handle.net/10316.2/35938

DOI:

DOI:http://dx.doi.org/10.14195/978-989-26-0738-2_16

Accessed : $\quad$ 26-Apr-2023 14:02:51

A navegação consulta e descarregamento dos títulos inseridos nas Bibliotecas Digitais UC Digitalis, UC Pombalina e UC Impactum, pressupõem a aceitação plena e sem reservas dos Termos e Condições de Uso destas Bibliotecas Digitais, disponíveis em https://digitalis.uc.pt/pt-pt/termos.

Conforme exposto nos referidos Termos e Condições de Uso, o descarregamento de títulos de acesso restrito requer uma licença válida de autorização devendo o utilizador aceder ao(s) documento(s) a partir de um endereço de IP da instituição detentora da supramencionada licença.

Ao utilizador é apenas permitido o descarregamento para uso pessoal, pelo que o emprego do(s) título(s) descarregado(s) para outro fim, designadamente comercial, carece de autorização do respetivo autor ou editor da obra.

Na medida em que todas as obras da UC Digitalis se encontram protegidas pelo Código do Direito de Autor e Direitos Conexos e demais legislação aplicável, toda a cópia, parcial ou total, deste documento, nos casos em que é legalmente admitida, deverá conter ou fazer-se acompanhar por este aviso.

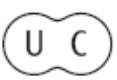




\section{INVESTIGAÇÃO OPERACIONAL EM AÇÃO \\ CASOS DE APLICAÇÃO}

RUI CARVALHO OLIVEIRA JOSÉ SOEIRO FERREIRA (EDITORES)

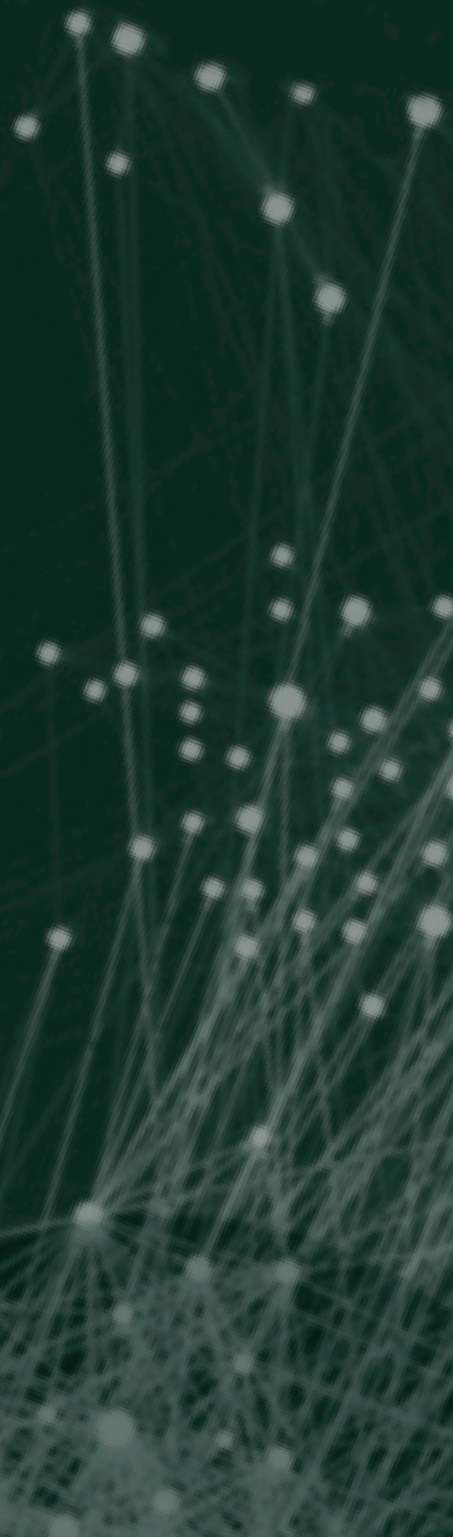




\section{CASO 16}

OTIMIZAÇÃO DA OPERAÇÃO DE REDES DE DISTRIBUIÇÃO: CONSIDERAÇÃO SIMULTÂNEA DE VARIÁVEIS TOPOLÓGICAS, TOMADAS DE TRANSFORMADORES E BATERIAS DE CONDENSADORES UTILIZANDO EPSO E HEURíSTICAS

Jorge Pereira INESC Porto, Faculdade de Economia Universidade do Porto jpereira@inescporto.pt

Jorge Alves INESC Porto, Faculdade de Engenharia Universidade do Porto pds11007@fe.up.pt

Manuel Matos INESC Porto, Faculdade de Engenharia Universidade do Porto mmatos@inescporto.pt 


\section{RESUMO}

A identificação de estratégias ótimas de exploração da rede tendo em conta a minimização da potência ativa de perdas assume uma relevância crescente na operação de distribuição de energia elétrica. Um novo método para esta identificação foi desenvolvido através da resolução do problema da determinação do ponto de operação ótimo, tendo como possibilidade a alteração da configuração da topologia de rede conjuntamente com a alteração das posições das tomadas dos transformadores e baterias de condensadores. O método de otimização EPSO modificado foi utilizado para resolver este problema.

\section{PALAVRAS-CHAVE}

Reconfiguração topológica, Voltage Var Control, OPF, EPSO, DMS 


\section{Introdução}

A operação das redes de distribuição de energia elétrica tem vindo a assumir uma importância cada vez maior, dado que é neste tipo de redes que se verifica a ocorrência de situações problemáticas tanto ao nível da qualidade de serviço, assim como em aspetos relacionados com a operação ótima do ponto de vista económico. Desta forma, a identificação de estratégias ótimas de exploração da rede tendo em conta a minimização da potência ativa de perdas assume uma relevância crescente. Um novo modelo para esta identificação foi desenvolvido e integrado num pacote de software comercial para gestão de redes da EFACEC, que está a ser instalado no novo Centro de Despacho da Região de Ática na Grécia.

O modo como as redes de distribuição são exploradas é fortemente determinado pela configuração da topologia em operação (em particular quando é imposta uma estrutura radial), pelas posições das tomadas nos transformadores e pelos escalões das baterias de condensadores colocados em serviço. Estes aspetos refletem-se sob diversas formas nas condições de exploração das redes e na qualidade de serviço oferecida aos consumidores. O problema relacionado com a determinação da configuração da topologia de rede ótima é geralmente considerado separadamente do problema da determinação das posições ótimas das tomadas dos transformadores e baterias de condensadores. O método de otimização proposto representa uma unificação destes dois problemas, uma vez que são tratados em simultâneo através da implementação de um algoritmo EPSO ("Evolutionary Particle Swarm Optimization") modificado, como é descrito nas secções seguintes.

A existência de produção distribuída de potência reativa, em diversos locais da rede, é um recurso que permite diminuir os trânsitos de potência reativa nos ramos das redes. Os ramos da rede poderão assim ser utilizados de forma mais completa para transmissão de potência ativa, evitando-se ou adiando-se assim novos investimentos. Por outro lado, a identificação da topologia em operação, assim como das tomadas de transformadores e dos escalões de baterias de condensadores, pode ser realizada especificando um conjunto alargado de restrições que deverão ser verificadas. Nestas restrições poderão ser consideradas: restrições associadas aos limites de intensidade de corrente em 
ramos; e restrições impondo gamas admissíveis para o módulo da tensão nos nós. A consideração das restrições para o módulo das tensões pode ser interpretada como a possibilidade de se permitir a especificação de variações máximas admissíveis para a tensão e, desta forma, inclui-se a especificação de quedas de tensão máximas admissíveis. Assim, há uma contribuição para garantir uma qualidade de serviço acrescida.

No caso de existirem geradores ligados à rede de distribuição (e não apenas ligações a redes de níveis de tensão mais elevados), decisões de despacho dessas máquinas podem também influenciar o resultado económico da exploração. A otimização global pode ser feita tendo em consideração um ou mais cenários de carga, podendo ainda incluir-se a otimização da transição entre cenários, com minimização do critério do número de manobras.

Se no problema de otimização for considerado que se podem ter avarias que conduzam a reconfigurações de emergência para restabelecimento de serviço, pode ainda ser acrescentado na formulação do problema um critério de minimização de potência cortada (mesmo sem reconfiguração) ou de potência não recuperada depois da reconfiguração. Esta hipótese é especialmente interessante se existirem cargas com diferentes graus de prioridade, correspondendo de certo modo às restrições de segurança do OPF (Optimal Power Flow - Trânsito de Potências Ótimo) para um EMS (Energy Management System - Sistema de Gestão das Redes de Transmissão). A reconfiguração para restabelecimento de serviço corresponde a um módulo de cálculo elétrico que é oferecida dentro de um DMS (Distribution Management System - Sistema de Gestão das Redes de Distribuição).

Nas próximas secções descreve-se a resolução do problema da determinação do ponto de operação ótimo, tendo como possibilidade a alteração da configuração da topologia de rede ótima conjuntamente com a alteração das posições das tomadas dos transformadores e baterias de condensadores. Assim, nas secções seguintes descrevem-se mais em detalhe os principais problemas existentes na otimização da operação de redes de distribuição, desenvolvendo-se uma formulação matemática para o problema, e dando-se saliência à incorporação de técnicas de Investigação Operacional, em particular algoritmos de otimização. Na secção 2 é descrito o problema da 
reconfiguração topológica, na secção 3 é apresentado o problema de controlo de tensão e potência reativa e finalmente na secção 4 é apresentada a metodologia proposta para a resolução conjunta dos dois problemas anteriores. Finalmente, na secção 5, são apresentados os resultados obtidos com a metodologia proposta para uma rede de teste.

\section{Reconfiguração Topológica}

Reconfiguração de redes é um problema de otimização em que se pretende otimizar o ponto de operação da rede em termos da minimização das perdas técnicas ou da melhoria dos perfis de tensão, dando como resultado sugestões de alterações na topologia da rede (também designada por configuração da rede).

As redes elétricas de distribuição são normalmente exploradas radialmente, mas têm uma estrutura que inclui malhas ou anéis (com alguns ramos normalmente abertos), que permite diferentes configurações de operação e aumenta a fiabilidade do sistema. A reconfiguração destas redes, através da alteração de estado dos aparelhos de corte, permite otimizar o funcionamento em face de um regime de carga, minimizando as perdas de energia e garantindo a satisfação das restrições técnicas (limites de carga dos ramos e queda de tensão nos nós).

Diversos autores dedicaram-se a estudar este problema, desenvolvendo e implementando diversas abordagens. Em 1975, MERLIN, A. e BACK, H. propuseram um método de otimização que utiliza uma técnica denominada por branch-and-bound para encontrar uma nova configuração admissível. CINVALAR, S. et al. (1988) por seu lado apresentam uma heurística baseada na estimação eficiente da redução das perdas ativas na rede, pela transferência de carga entre duas saídas de uma subestação de distribuição.

Mais recentemente têm sido aplicadas técnicas de otimização baseadas em meta-heurísticas, como algoritmos genéticos GUPTA, N. et al. (2010), simmulated annealing EL-HAWARY, M. (1998), e métodos baseados em populações de soluções como o Particle Swarm Optimization GUPTA, N., SWARNKAR, A., NIAZI, K.R. (2011). 
Em termos de reconfiguração topológica, para além do problema de otimização da operação, há ainda um problema adicional relacionado com o restauro de serviço na sequência de uma avaria, onde a reconfiguração visa permitir realimentar pelo menos parte dos consumidores que ficaram privados de eletricidade, através de percursos alternativos. Em ambos os problemas descritos, a minimização do número de manobras (ações de abertura/fecho de aparelhos de corte) pode ser também um critério adicional a considerar.

Um algoritmo desenvolvido e integrado num DMS, por MATOS, M.A., MELO, P. (1999), para lidar com estes problemas de reconfiguração baseia-se numa heurística fundada num processo de substituição de ramos (branch exchange) onde a seleção de ramos a fechar e abrir é realizada a partir de valores locais de tensão nos nós e correntes nos ramos adjacentes. Este algoritmo utilizou uma meta-heurística de otimização, o simulated annealing, sendo as vizinhanças definidas pela abertura/fecho de ramos (de modo a manter a rede radial).

Na referência KHODR, H.M. et al. (2009), o objetivo e as restrições deste problema foram incluídos no problema de controlo de tensão e potência reativa (descrito na secção seguinte), tendo sido assim reunidos num problema único, e que foi designado por DOPF (Distribution Optimal Power Flow). Nesta referência, foi feita uma pesquisa bibliográfica detalhada sobre o problema de reconfiguração topológica, e concluiu-se que o problema de reconfiguração ótima, não foi apresentado ou estudado ao mesmo tempo e no mesmo modelo que o problema controlo de tensão e potência reativa. Uma abordagem a este problema integrado é descrita na secção 4.

\section{Controlo de tensão e potência reativa}

A otimização do funcionamento de sistemas elétricos de energia também está relacionada com a posição das tomadas de baterias de condensadores e a posição de tomadas de transformadores com regulação em carga. Com a alteração da quantidade de potência reativa injetada pela bateria de condensadores pode-se fazer subir ou baixar a tensão numa determinada zona da rede onde a bateria está localizada. Com a alteração da razão de transformação de um transformador pode-se fazer subir ou baixar a tensão na 
zona da rede ligada ao secundário do transformador. No caso de existirem geradores síncronos ligados à rede, que permitem controlar a tensão dos nós a que estão ligados, também há influência no resultado económico da exploração por causa da tensão em que é colocado o respetivo barramento.

Várias abordagens a este problema têm sido sugeridas ao longo dos anos por diferentes autores. Em LIU, Y., ZHANG, P., QIU, X. (2002), foi utilizada programação dinâmica em conjunto com um sistema de lógica difusa para controlar a tensão à saída da subestação. Em HSU, Y. (1998), foi considerado o problema do controlo de tensão multitemporal, para as 24 horas seguintes. Foi utilizada uma rede neuronal e programação dinâmica difusa para resolver este problema multitemporal, tendo por base heurísticas definidas com base na experiência passada dos operadores de rede.

Outros trabalhos sugerem a ideia de utilizar plataformas de inferência difusa para corrigir desvios de tensão e controlo de potência reativa JUNGES, M. (2000) e introduzem conceitos baseados em lógica difusa para o controlo de sistemas e subsistemas de energia elétrica EKEL, P. et al. (1998). Exemplos de aplicação de controladores difusos em problemas de estabilidade podem ser encontrados em LIU, C.W., CHANG, C.S., JIANG, J.A. (2001). LIANG, R. (2003) apresenta uma metodologia baseada em simulated annealing que, em conjunto com uma estratégia difusa de controlo de potência reativa, é aplicada ao problema de determinar o escalonamento ótimo das tomadas de baterias de condensadores e transformadores, para um sistema de distribuição.

A utilização de conceitos baseados em lógica difusa para o controlo de sistemas de energia elétrica surge como alternativa ou complemento ao uso de métodos como o simulated annealing PEREIRA, J., SARAIVA, J.T., PONCE DE LEÃO, M.T. (1999), algoritmos evolucionários MIRANDA, V., FONSECA, N. (2002) ou outras meta-heurísticas MIRANDA, V., CALISTO, P. (2002) cujo principal objetivo é o de derivar ações de controlo para controlar tensões e reduzir perdas, visto que apresenta uma eficiência muito mais elevada em termos de esforço computacional.

A resolução deste problema é um dos módulos integrantes de um DMS, cujo objetivo é controlar tensões em redes que possuam diversos tipos de equipamentos que permitam a regulação em carga. Este módulo é 
habitualmente designado por Voltage Var Control - Controlo de tensão e potência reativa.

A função objetivo deste problema de otimização representa as perdas técnicas da rede (1), e resolve-se como um problema de minimização. As restrições do problema estão relacionadas com: a satisfação das equações do problema do balanço de potências em cada nó (2) - primeira lei de Kirchhoff e respetivo cálculo dos trânsitos de potência nas linhas - segunda lei de Kirchhoff -; a manutenção das tensões em todos os nós da rede dentro de uma banda admissível (3); e a garantia que o trânsito de potência em todas as linhas da rede não exceda os seus limites térmicos (4). Este problema é um problema de otimização não linear misto, com as variáveis inteiras representadas em (5) e (6).

$\operatorname{Min} \mathrm{Z}=\sum_{\mathrm{ij}}-\mathrm{G}_{\mathrm{ij}}\left(\mathrm{V}_{\mathrm{i}}^{2}+\mathrm{V}_{\mathrm{j}}^{2}-2 \mathrm{~V}_{\mathrm{i}} \mathrm{V}_{\mathrm{j}} \cos \left(\theta_{\mathrm{i}}-\theta_{\mathrm{j}}\right)\right)$

sujeito a:

$\mathrm{g}\left(\mathrm{V}_{\mathrm{i}}, \theta_{\mathrm{i}}, \mathrm{t}_{\mathrm{f}}, \mathrm{e}_{\mathrm{c}}\right)=0$

$\mathrm{V}^{\min } \leq \mathrm{V}_{\mathrm{i}} \leq \mathrm{V}^{\mathrm{max}}$ para cada nó

$\left|\mathrm{I}_{\mathrm{ij}}\left(\mathrm{V}_{\mathrm{i}}, \theta_{\mathrm{i}}, \mathrm{V}_{\mathrm{j}}, \theta_{\mathrm{j}}\right)\right| \leq\left|\mathrm{I}_{\mathrm{ij}}\left(\mathrm{V}_{\mathrm{i}}, \theta_{\mathrm{i}}, \mathrm{V}_{\mathrm{j}}, \theta_{\mathrm{j}}\right)\right|^{\mathrm{max}}$ para cada ramo ij

$\mathrm{t}_{\mathrm{f}} \in\left\{\mathrm{t}_{\mathrm{f}}^{1}, \mathrm{t}_{\mathrm{f}}^{2}, \ldots, \mathrm{t}_{\mathrm{f}}^{\mathrm{k}_{\mathrm{f}}}\right\}$ para cada transformador $\mathrm{f}$ com kf tomadas

$\mathrm{e}_{\mathrm{c}} \in\left\{\mathrm{e}_{\mathrm{c}}^{1}, \mathrm{e}_{\mathrm{c}}^{2}, \ldots, \mathrm{e}_{\mathrm{c}}^{\mathrm{k}_{\mathrm{c}}}\right\}$

para cada bateria de condensadores c com kc secções

$$
i, j \in\{1,2, \ldots, n b\}
$$

Nesta formulação:

- gO representa as equações do problema de trânsito de potências;

- $\mathrm{t}_{\mathrm{f}}$ representa um dos valores disponíveis de tomadas de transformadores;

- e $_{c}$ representa um dos valores disponíveis de secções de baterias de condensadores;

- $V_{\mathrm{i}}$ e $\theta_{\mathrm{i}}$ representam o módulo e a fase da tensão em cada nó da rede;

- $\mathrm{G}_{\mathrm{ij}}$ corresponde à parte real da matriz das admitâncias nodais (característica física dos ramos da rede); 
- $\left|\mathrm{I}_{\mathrm{ij}}\left(\mathrm{V}_{\mathrm{i}}, \theta_{\mathrm{i}}, \mathrm{V}_{\mathrm{j}}, \theta_{\mathrm{j}}\right)\right|$ representa o módulo da intensidade de corrente no ramo que liga o nó i ao nó j;

- nb representa o número de barramentos da rede;

Este problema pode ser resolvido através de um sistema de controlo de tensões como o que é apresentado em MIRANDA, V., CALISTO, P. (2002) e MIRANDA, V., MOREIRA, A., PEREIRA, J. (2007), sendo um procedimento eficiente em termos de tempo de execução necessário para encontrar uma solução viável. Este controlador demonstra grande superioridade face a outros tipos de controlo que utilizam algoritmos computacionalmente mais pesados. É frequente que estes tipos de algoritmos de controlo possam necessitar de centenas a milhares de iterações, enquanto o controlador desenvolvido necessita apenas de um reduzido número de iterações para atingir um resultado viável.

MIRANDA, V., MOREIRA, A., PEREIRA, J. (2007) descrevem uma arquitetura para o módulo Voltage Var Control que tem por base uma cascata de controladores difusos do tipo Mamdani, que constituem um sistema de inferência baseado em lógica difusa (ver Figura 1), e uma rotina de trânsitos de potência que avalia, periodicamente, o resultado de todas as ações propostas. As regras difusas apresentadas estão relacionadas com a eficiência de uma ação de controlo, bem como com a posição atual de cada um dos diferentes equipamentos utilizados no controlo.

Além do principal objetivo de manter as tensões em todos os nós da rede dentro de uma banda admissível, é da responsabilidade do controlador difuso garantir que o trânsito de potências em todas as linhas da rede não exceda os limites térmicos. Este controlador difuso será utilizado no final do processo de otimização apresentado na secção seguinte para dar mais garantias ao algoritmo da solução encontrada ser mais próxima da ótima.

\section{Reconfiguração com controlo de tensão e potência reativa}

O algoritmo para resolver o problema do DOPF deverá ser capaz de encontrar a configuração ótima de operação de um sistema de distribuição de energia elétrica, para um cenário de operação, avaliando a carga distribuída 
pelas subestações e pelas saídas destas. A solução final deverá ser uma solução admissível, onde todas as cargas deverão estar alimentadas e os limites técnicos dos componentes do sistema deverão ser cumpridos. Este é um problema com um grande número de soluções alternativas, sendo que o algoritmo deverá escolher aquela que otimiza a função objetivo escolhida.

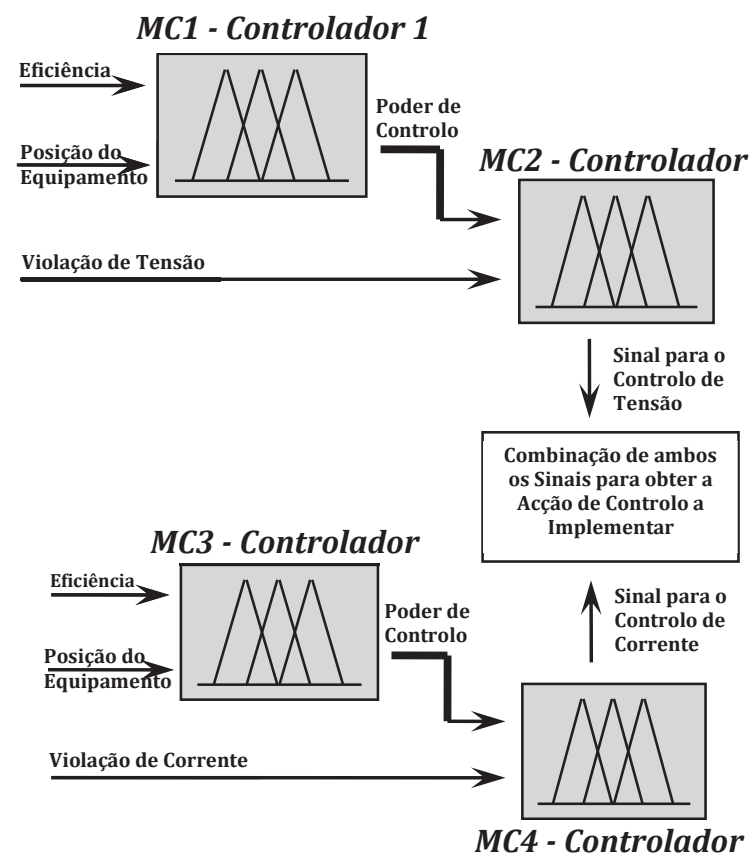

Figura 1: Esquema do sistema de inferência baseado em lógica difusa para controlo de tensão e potência reativa

Na secção anterior apresentou-se a formulação do problema de controlo de tensão e potência reativa, onde a função objetivo é a minimização das perdas na rede (1), com restrições relativas ao balanço de potência nos nós - primeira lei de Kirchhoff -; cálculo de tensões nos nós da rede - segunda lei de Kirchhoff -; limites de trânsito de potência nas linhas; limites de máximo e mínimo de tensão; e limites de tomadas de transformadores e baterias de condensadores associados a variáveis inteiras. 
A formulação completa do problema integrado de reconfiguração com controlo de tensão e potência reativa, com a função objetivo e as restrições, é apresentada nos seguintes parágrafos.

Função objetivo de minimização das perdas técnicas da rede:

$$
\text { Min } P_{\text {perdas }}^{\text {total }}=\sum_{\mathrm{ij}}-\mathrm{G}_{\mathrm{ij}}\left(\mathrm{y}_{\mathrm{ij}}\right)\left(\mathrm{V}_{\mathrm{i}}^{2}+\mathrm{V}_{\mathrm{j}}^{2}-2 \mathrm{~V}_{\mathrm{i}} \mathrm{V}_{\mathrm{j}} \cos \left(\theta_{\mathrm{i}}-\theta_{\mathrm{j}}\right)\right)
$$

Sujeito a:

Limites técnicos dos trânsitos de potência nos ramos (linhas e transformadores):

$$
S_{i j}^{\text {min. }} \leq S_{i j}\left(V_{i}, \theta_{i}, V_{j}, \theta_{j}\right) \leq S_{i j}^{\max }
$$

Limites técnicos de tensão nos nós da rede:

$$
\mathrm{V}_{\mathrm{i}}^{\text {min. }} \leq \mathrm{V}_{\mathrm{i}} \leq \mathrm{V}_{\mathrm{i}}^{\max }
$$

Condição de radialidade, que consiste em verificar que o número de linhas fora de serviço no início deve ser igual no final do processo, com todos os consumidores abastecidos:

$$
\begin{gathered}
\sum_{\mathrm{ij}}\left(1-\mathrm{y}_{\mathrm{ij}}\right)=\mathrm{N}_{\text {abertos }}^{\text {inic. }} \\
\mathrm{S}_{\mathrm{L}}^{\text {total }}=\mathrm{S}_{\mathrm{L}}^{\text {inicial }} \\
\operatorname{real}\left(\mathrm{S}_{\mathrm{L}}^{\text {total }}\right)=\sum_{\mathrm{i}} \mathrm{P}_{\mathrm{i}}^{\mathrm{L}} \\
\operatorname{imag}\left(\mathrm{S}_{\mathrm{L}}^{\text {total }}\right)=\sum_{\mathrm{i}} \mathrm{Q}_{\mathrm{i}}^{\mathrm{L}}
\end{gathered}
$$

Limites máximos e mínimos de potência produzida nos geradores:

$$
\mathrm{S}_{\mathrm{Gi}}^{\min } \leq \mathrm{S}_{\mathrm{Gi}} \leq \mathrm{S}_{\mathrm{Gi}}^{\max }
$$

Limites de tomadas nos transformadores:

$$
\mathrm{t}_{\mathrm{f}}^{1} \leq \mathrm{t}_{\mathrm{f}} \leq \mathrm{t}_{\mathrm{f}}^{\mathrm{k}_{\mathrm{f}}}
$$

Limites de tomadas nas baterias de condensadores:

$$
\mathrm{e}_{\mathrm{c}}^{1} \leq \mathrm{e}_{\mathrm{c}} \leq \mathrm{e}_{\mathrm{c}}^{\mathrm{k}_{\mathrm{c}}}
$$


Balanço de potência na rede, a produção tem de ser igual ao consumo:

$$
\begin{gathered}
S_{\mathrm{L}}^{\text {total }}+\mathrm{S}_{\text {perdas }}^{\text {total }}=\mathrm{S}_{\mathrm{G}}^{\text {total }} \\
\operatorname{real}\left(\mathrm{S}_{\mathrm{G}}^{\text {total }}\right)=\sum_{\mathrm{i}} \mathrm{P}_{\mathrm{i}}^{\mathrm{G}} \\
\operatorname{imag}\left(\mathrm{S}_{\mathrm{G}}^{\text {total }}\right)=\sum_{\mathrm{i}} \mathrm{Q}_{\mathrm{i}}^{\mathrm{G}} \\
\operatorname{real}\left(\mathrm{S}_{\text {perdas }}^{\text {total }}\right)=\mathrm{P}_{\text {perdas }}^{\text {total }} \\
\operatorname{imag}\left(\mathrm{S}_{\text {perdas }}^{\text {total }}\right)=\sum_{\mathrm{ij}} \mathrm{B}_{\mathrm{ij}}\left(\mathrm{y}_{\mathrm{ij}}\right)\left(\mathrm{V}_{\mathrm{i}}^{2}+\mathrm{V}_{\mathrm{j}}^{2}-2 \mathrm{~V}_{\mathrm{i}} \mathrm{V}_{\mathrm{j}} \cos \left(\theta_{\mathrm{i}}-\theta_{\mathrm{j}}\right)\right)
\end{gathered}
$$

Equações do trânsito de potência ativa nos ramos e balanço nulo num nó:

$$
P_{i}^{G}-P_{i}^{L}=V_{i} \sum_{j} V_{j}\left(G_{i j}\left(y_{i j}\right) \cos \left(\theta_{i}-\theta_{j}\right)+B_{i j}\left(y_{i j}\right) \sin \left(\theta_{i}-\theta_{j}\right)\right)
$$

Equações do trânsito de potência reativa nos ramos e balanço nulo num nó:

$$
\begin{gathered}
Q_{i}^{G}-Q_{i}^{L}=V_{i} \sum_{j} V_{j}\left(G_{i j}\left(y_{i j}\right) \sin \left(\theta_{i}-\theta_{j}\right)-B_{i j}\left(y_{i j}\right) \cos \left(\theta_{i}-\theta_{j}\right)\right) \\
G_{i j}\left(y_{i j}\right)=\left\{\begin{array}{cl}
G_{i j} & , \text { se } y_{i j}=1 \\
0 & , \text { se } y_{i j}=0
\end{array}\right. \\
B_{i j}\left(y_{i j}\right)=\left\{\begin{array}{cl}
B_{i j} & , \text { se } y_{i j}=1 \\
0 & , \text { se } y_{i j}=0
\end{array}\right.
\end{gathered}
$$

As variáveis de decisão são:

- $\mathrm{t}_{\mathrm{f}}$ - variável inteira de decisão associada à tomada do transformador $\mathrm{f}$

- $\mathrm{e}_{\mathrm{c}}$ - variável inteira de decisão associada à tomada da bateria de condensadores C

- $y_{\mathrm{ij}}$ - variável binária de decisão relativa à configuração topológica do ramo ij (1 - ramo ij em serviço, 0 - ramo ij fora de serviço)

Nesta formulação:

- $\mathrm{S}_{\mathrm{G}}^{\text {total }}$ - total de potência aparente gerada

- $\mathrm{S}_{\mathrm{L}}^{\text {total }}$ - total de potência aparente consumida

- $\mathrm{S}_{\mathrm{ij}}^{\mathrm{min}}$ - mínimo de potência aparente no ramo $\mathrm{ij}$

- $\mathrm{S}_{\mathrm{ij}}^{\max }$ - máximo de potência aparente no ramo ij

- $\mathrm{V}_{\mathrm{i}}^{\text {min. }}$ - valor mínimo para o módulo da tensão no nó i

- $\mathrm{V}_{\mathrm{i}}^{\max }$ - valor máximo para o módulo da tensão no nó i

- $\quad \mathrm{N}_{\text {abertos }}^{\text {inic. }}$ - número de linhas fora de serviço na configuração inicial

- $\mathrm{S}_{\mathrm{Gi}}^{\mathrm{min}}$ - potência aparente mínima no gerador $\mathrm{i}$ 
- $\mathrm{S}_{\mathrm{Gi}}^{\max }$ - potência aparente máxima no gerador $\mathrm{i}$

- $\mathrm{t}_{\mathrm{f}}^{1}$ e $\mathrm{t}_{\mathrm{f}}^{\mathrm{k}_{\mathrm{f}}}$ - tomada 1 (menor valor das tomadas) e tomada $\mathrm{kf}$ (maior valor das tomadas) do transformador $\mathrm{f}$ com $\mathrm{kf}$ tomadas

- $\mathrm{e}_{\mathrm{c}}^{1}$ e $\mathrm{e}_{\mathrm{c}}^{\mathrm{k}_{\mathrm{c}}}$ - tomada 1 (menor valor das tomadas) e tomada kc (maior valor das tomadas) da bateria de condensadores c com kc secções

- $\mathrm{V}_{\mathrm{i}}$ - módulo da tensão no nó i

- $\theta_{\mathrm{i}}$ - fase da tensão no nó i

- $\mathrm{G}_{\mathrm{ij}}$ - elemento da parte real da matriz das admitâncias nodais

- $\mathrm{B}_{\mathrm{ij}}$ - elemento da parte imaginária da matriz das admitâncias nodais

- $\mathrm{P}_{\mathrm{i}}^{\mathrm{G}}$ - potência ativa gerada no nó i

- $\mathrm{Q}_{\mathrm{i}}^{\mathrm{G}}$ - potência reativa gerada no nó i

- $\mathrm{P}_{\mathrm{i}}^{\mathrm{L}}$ - potência ativa consumida no nó i

- $\mathrm{Q}_{\mathrm{i}}^{\mathrm{L}}$ - potência reativa consumida no nó i

- $\mathrm{S}_{\mathrm{L}}^{\text {inicial }}$ - valor da potência aparente consumida, no estado inicial da rede

A principal diferença entre a função objetivo (1) e (8) reside na variável de decisão relacionada com a topologia, $\mathrm{y}_{\mathrm{ij}}$, que terá apenas dois valores possíveis: 0, se o ramo ij está fora de serviço; e 1, se o ramo ij está em serviço. Desta forma, a parte real da matriz das admitâncias nodais, $G_{i j}$, será igual a zero no caso do ramo estar fora de serviço e sendo, por isso, eliminada do cálculo das perdas globais. Por outro lado, se o ramo estiver em serviço, ele será considerado para o cálculo das perdas, pois nesta situação $G_{i j}$ tem o valor característico do ramo ij.

O método de resolução do problema proposto admite a possibilidade de existência de anéis fechados na rede, por exemplo entre duas saídas de uma mesma subestação, ou entre duas subestações diferentes. Caso existam estes anéis fechados na rede, o algoritmo trata-os como zonas não otimizáveis, garantindo a operação radial nas restantes zonas da rede, sujeitas a otimização.

O algoritmo implementado para resolver este problema parte de uma configuração topológica inicial, e a partir desta faz uma pesquisa para encontrar a configuração ótima, gerando um conjunto de configurações alternativas que se movem no espaço de soluções por meio de um operador. Todas as configurações que não pertençam ao espaço admissível de soluções 
por violação de limites técnicos são penalizadas de forma a não serem escolhidas.

Foi utilizado o algoritmo EPSO MIRANDA, V., FONSECA, N. (2002) para resolver este problema, conjuntamente com algumas heurísticas locais que têm em conta o conhecimento que existe sobre as relações existentes entre as variáveis de decisão e os seus efeitos nas restrições. Estas heurísticas têm a função de garantir a admissibilidade topológica das soluções encontradas e ajustes de variáveis relacionadas com o controlo de tensão na rede, fazendo com que configurações, que à partida poderiam ser penalizadas por não satisfação dos critérios de tensão ou dos limites nos ramos, possam ser consideradas como admissíveis por alteração de algumas variáveis de controlo relativas ao módulo da tensão.

O algoritmo EPSO foi proposto por Miranda e Fonseca em 2002 como um novo algoritmo de otimização que explora as fraquezas do algoritmo PSO (Particle Swarm Optimization) já existente KENNEDY, J., EBERHART, R. (1995). O EPSO utiliza os mesmos operadores que o PSO, mas no primeiro os pesos dos operadores internos do método são também otimizados, utilizando conceitos evolucionários. Ambas as estratégias de otimização são estratégias populacionais, ou seja, criam um conjunto de soluções, denominadas de partículas, que se movem pelo espaço de soluções de acordo com as respetivas posições passadas e a melhor posição encontrada até ao momento, do conjunto de partículas (enxame).

O operador de movimento que faz mover cada partícula possui três termos, cada um com o respetivo peso: inércia - relacionado com as posições passadas da partícula; memória - relacionado com a melhor posição passada da partícula; cooperação - relacionado com a melhor posição encontrada pelo enxame. São os pesos de cada um destes termos, que no algoritmo EPSO, são otimizados utilizando o conceito de mutação dos algoritmos evolucionários.

As modificações introduzidas no algoritmo EPSO para o adaptar ao problema aqui descrito baseiam-se no facto da convergência do método ser acelerada através da utilização de heurísticas simples, que permitem gerar, na maioria dos casos, soluções admissíveis. Desta forma, no início do processo é criado um grafo da rede, que a simplifica e permite a identificação dos ramos 
que pertencem a cada anel fundamental da rede. As heurísticas implementadas relacionadas com configuração da rede não permitem que mais do que um ramo se encontre fora de serviço em cada anel, e que cada ramo a abrir pertença a um anel da rede.

Por outro lado, no que diz respeito ao controlo de tensão, as tomadas dos transformadores e das baterias de condensadores são ajustadas caso haja violação dos limites de tensão ou dos limites dos ramos. Nas redes de distribuição de energia elétrica o controlo do módulo da tensão é efetuado através de transformadores e de baterias de condensadores. Estes equipamentos possuem tomadas que podem ser ajustadas de modo a manter o módulo da tensão, no nó de controlo, num determinado valor especificado, sendo portanto um controlo discreto da tensão. Ao controlar e equilibrar os módulos da tensão nos nós da rede, os fluxos de energia na rede são diminuídos, baixando-se assim os trânsitos de potência nos ramos.

Após a execução do EPSO para reconfigurar a rede de forma integrada é executado um algoritmo de controlo de tensão e potência reativa [18] que faz os ajustes finais das tomadas de controlo, obtendo-se assim uma solução bastante boa para o problema.

\section{Exemplo}

Para testar o algoritmo descrito na secção anterior, foi considerada a rede de teste apresentada na Figura 2, que corresponde a uma versão simplificada de uma rede de distribuição de energia elétrica. A rede de teste apresentada é uma versão modificada da rede utilizada em BHOOMESH, R., AH KING, R.T.F., RUGHOOPUTH, H.C.S. (2003), sendo constituída por 3 feeders (saídas de subestações), 3 transformadores (T1, T2 e T3), 19 nós (F1, F2, F3, 1 a 16), 20 linhas (L1 a L20), 13 cargas elétricas (correspondentes a Postos de Transformação onde se liga a rede de Baixa Tensão ou correspondentes a Consumidores de Média Tensão) e 7 baterias de condensadores (C1 a C7). As linhas possuem um aparelho de corte que através da sua abertura inibe a passagem de corrente elétrica pela linha. Cada uma das referidas saídas pode corresponder a três saídas de uma mesma subestação ou três saídas de três subestações diferentes. 
A montante dos nós F1, F2 e F3 está a rede de transporte e os geradores de um sistema elétrico convencional. As cargas estão representadas por setas nos nós de consumo e o seu valor total é 28,6 MW. Refira-se que o cenário de operação da rede é caracterizado pelo consumo em cada um dos nós de consumo da rede, sendo a operação da rede otimizada para este cenário de consumo.

A configuração inicial da rede é a que está apresentada na Figura 2, onde os ramos L6, L8, L12, L17, L18, L19 e L20 estão abertos. Para alterar a configuração da rede existem aparelhos de corte em cada ramo, que permitem ligar ou desligar o respetivo ramo. De notar que os ramos que se encontram abertos fecham caminhos entre as diferentes saídas, o que permite transferir carga de uma saída para outra. Obviamente, esta transferência de carga está limitada pela capacidade de cada uma das saídas.

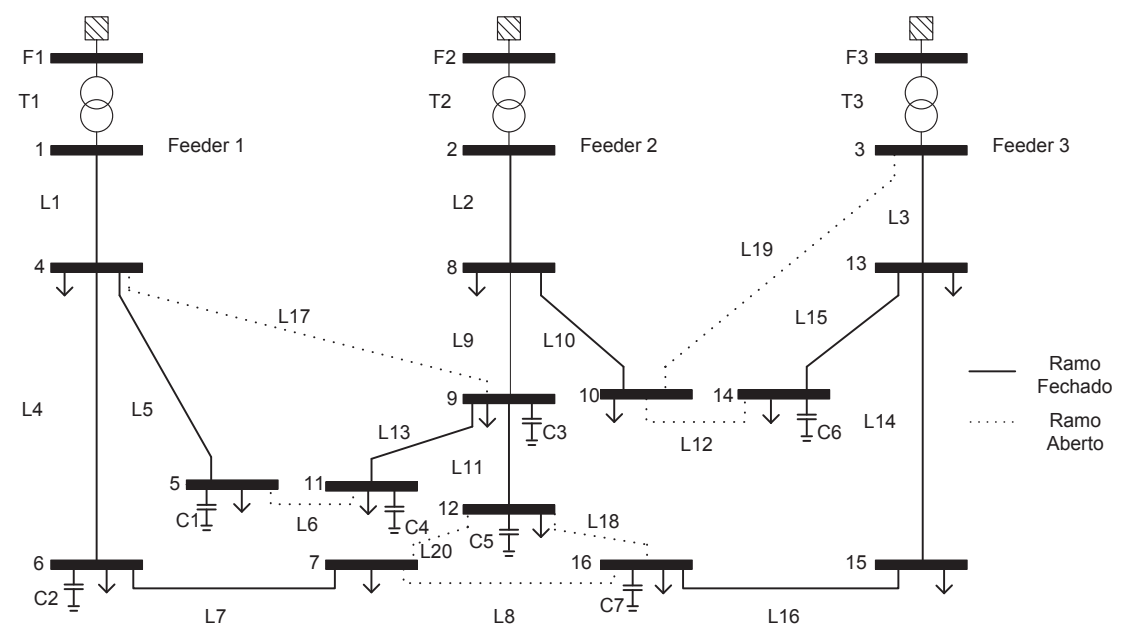

Figura 2: Rede de distribuição com possibilidade de reconfiguração.

O algoritmo EPSO proposto para a reconfiguração ótima da rede foi configurado com os seguintes parâmetros: uma população com 45 indivíduos; a probabilidade de comunicação é de 0,2; o parâmetro de mutação igual a 0,8; e o critério de paragem utilizado foi definido como o número máximo de iterações e igual a 35. 
A otimização da rede, relativamente às perdas de energia elétrica, foi obtida através de quatro modos de otimização diferentes: reconfiguração e controlo de tensão (CT) integrados (Reconfiguração e CT); reconfiguração seguida do controlo de tensão (Reconfiguração + CT, resolução separada e tradicional); só reconfiguração (Reconfiguração); só controlo de tensão (CT).

Esta otimização foi realizada para um cenário de operação da rede, caracterizado pelo nível de carga em cada um dos nós. Os resultados estão apresentados na Tabela 1, e mostram que a utilização combinada dos módulos de reconfiguração e controlo de tensão é preferível pois conduz ao menor valor de perdas finais na rede (uma redução de 31\% relativamente às perdas iniciais), para o mesmo cenário de operação. Por outro lado quando o modo de operação considera a execução separada das operações de reconfiguração e das operações de controlo de tensão apenas conduz a uma redução das perdas de cerca de 29\%. Estes resultados mostram a vantagem do método proposto relativamente ao método tradicional no qual as operações de reconfiguração e controlo de tensão são consideradas separadamente.

\begin{tabular}{|c|c|c|c|c|}
\hline $\begin{array}{l}\text { Modo do } \\
\text { algoritmo }\end{array}$ & $\begin{array}{l}\text { Perdas } \\
\text { iniciais } \\
(\mathrm{kW})\end{array}$ & $\begin{array}{l}\text { Perdas } \\
\text { finais } \\
(\mathrm{kW})\end{array}$ & Equipamentos alterados & $\begin{array}{l}\text { Tempo de } \\
\text { execução } \\
\text { (s) }\end{array}$ \\
\hline $\begin{array}{c}\text { Reconfiguração } \\
\text { e CT }\end{array}$ & \multirow{4}{*}{335,5} & 230,1 & $\begin{array}{l}\text { F1, F2, F3, C1, C2, C3, C4, C5, } \\
\text { C6, C7, T2, T3, swit10, swit11, } \\
\text { swit13, swit15, swit06, swit12, } \\
\text { swit18, swit19 }\end{array}$ & 0,41 \\
\hline $\begin{array}{c}\text { Reconfiguração } \\
+ \text { CT }\end{array}$ & & 237,7 & $\begin{array}{l}\text { F1, F2, F3, C1, C2, C3, C4, C5, } \\
\text { C6, C7, T1, T2, T3, } \\
\text { swit10, swit11, swit19, swit20 }\end{array}$ & 0,7 \\
\hline Reconfiguração & & 313,7 & swit10, swit11, swit19, swit20 & 0,41 \\
\hline CT & & 246,9 & $\begin{array}{l}\mathrm{F} 1, \mathrm{~F} 2, \mathrm{~F} 3, \mathrm{~T} 1, \mathrm{~T} 2, \mathrm{~T} 3, \mathrm{C} 1, \mathrm{C} 2, \\
\mathrm{C} 3, \mathrm{C} 4, \mathrm{C} 5, \mathrm{C} 6, \mathrm{C} 7\end{array}$ & 0,01 \\
\hline
\end{tabular}

Tabela 1: Resultados da execução dos módulos de reconfiguração e controlo de tensão integrados, sequencialmente, e isoladamente cada um.

As operações topológicas efetuadas pelo algoritmo sugerem um alívio de carga do ramo central da rede para os periféricos. Isto é devido ao facto de as cargas situadas nos nós 9 e 12 serem cargas com valores mais elevados quando comparadas com as restantes cargas da rede, o que faz com que os ramos 
centrais se encontrem mais carregados e por isso com maiores perdas. Assim, o algoritmo procura aliviar estes ramos de forma a reduzir as perdas globais do sistema.

Notar que apesar de todos os aparelhos com capacidade de controlo de tensão, baterias de condensadores, equivalentes de rede e transformadores, serem todos operados nos quatro diferentes modos de execução do algoritmo de otimização, os estados finais de cada um destes equipamentos não é o mesmo.

Os tempos de execução da Tabela 1 e Tabela 2 foram obtidos num computador com um processador Intel® Core i7 CPU 3,40GHz e com 8 GB de memória RAM. Analisando os tempos de execução da Tabela 1, o modo integrado apresenta um tempo de execução de 0,41 segundos, que é um tempo inferior ao modo sequencial e semelhante ao modo de apenas reconfiguração, e conseguindo uma solução melhor.

\begin{tabular}{|c|c|c|c|}
\hline Modo do algoritmo & $\begin{array}{c}\text { Perdas iniciais } \\
(\mathrm{kW})\end{array}$ & $\begin{array}{c}\text { Perdas finais } \\
(\mathrm{kW})\end{array}$ & $\begin{array}{c}\text { Tempo de } \\
\text { execução (s) }\end{array}$ \\
\hline Reconfiguração e CT & \multirow{4}{*}{42561,8} & 35007,0 & 223,8 \\
\hline Reconfiguração + CT & & 35878,5 & 412,55 \\
\hline Reconfiguração & & 42471,8 & 184,4 \\
\hline CT & & 36033,4 & 44,0 \\
\hline
\end{tabular}

Tabela 2 - Resultados da execução dos módulos de reconfiguração e controlo de tensão integrados, sequencialmente, e isoladamente para uma rede real.

Para validar o bom comportamento do algoritmo desenvolvido em redes reais e de maior dimensão, na Tabela apresentam-se os resultados para uma rede de distribuição real, que é constituída por 3909 nós e 3914 ramos. Nesta rede continua a verificar-se um melhor desempenho em termos de solução encontrada por parte do modo integrado, conseguindo a melhor solução em termos do mínimo de perdas. Neste caso, o tempo de execução do modo integrado é bastante inferior ao modo de execução sequencial, obtendo a solução em tempo útil.

O algoritmo proposto para a reconfiguração ótima de rede com base na estratégia de otimização EPSO, com o objetivo de minimização das perdas 
ativas na rede, provou ser uma metodologia válida para lidar com este problema de uma forma mais eficiente.

\section{Conclusões}

O modelo desenvolvido revela-se um procedimento extremamente eficiente que resolve de forma integrada dois problemas que habitualmente são tratados de forma independente.

O exemplo apresentado neste artigo confirma os resultados obtidos por outros investigadores que aplicaram outros conceitos para a reconfiguração da rede e para o controlo de tensões e minimização de perdas em sistemas de energia elétrica de forma separada. No entanto, quando a resolução é feita de forma integrada, conseguem-se obter melhores resultados e de uma forma eficiente.

A utilização do EPSO para resolver este problema, conjuntamente com algumas heurísticas locais que têm em conta as relações existentes entre as variáveis, permitiu definir um algoritmo que resolve em tempo útil este problema. Quando comparado com outros algoritmos, que fazem apenas reconfiguração, o tempo total é da mesma ordem de grandeza.

Uma versão comercial deste algoritmo foi integrada num DMS comercial da EFACEC, estando atualmente a ser instalado em alguns dos seus clientes.

\section{Agradecimentos}

Este trabalho é parcialmente financiado por Fundos FEDER através do Programa Operacional Fatores de Competitividade - COMPETE e por Fundos Nacionais através da FCT - Fundação para a Ciência e a Tecnologia no âmbito do projeto GEMS PTDC/EEA-EEL/105261/2008.

\section{BibliografiA}

MERLIN, A., BACK, H. (1975) "Search for a minimum-loss operating spanning tree configuration in an urban power distribution", Proceedings of Fifth Power System Computation Conference, Cambridge, UK, pp. 1 - 18.

CINVALAR, S., GRAINGER, J.J., YIN, H., LEE, S.S.H. (1988) "Distribution feeder reconfiguration for loss reduction", IEEE Transactions on Power Delivery, vol. 3, no. 3, pp. 1217 - 1223. 
GUPTA, N., SWARNKAR, A., NIAZI, K.R., BANSAL, R.C. (2010) "Multi-objective reconfiguration of distribution systems using adaptive genetic algorithm in fuzzy framework", IET Generation, Transmission \& Distribution, vol. 4, no. 12, pp. 1288-1298, 2010.

EL-HAWARY, M. (editor) (1998) Electric Power Applications of Fuzzy Systems, IEEE Press Series on Power Engineering.

GUPTA, N., SWARNKAR, A., NIAZI, K.R. (2011) "Reconfiguration of Distribution Systems for Real Power Loss Minimization Using Adaptive Particle Swarm Optimization", Electric Power Components and Systems, vol. 39, no. 4, pp. 317 - 330.

MATOS, M.A., MELO, P. (1999) "Multi-objective Reconfiguration for Loss Reduction and Service Restoration Using Simulated Annealing”, Proceedings of IEEE Power Tech'99, Budapest, Hungary.

KHODR, H.M., MARTÍNEZ-CRESPO, J., MATOS, M.A., PEREIRA, J. (2009) "Distribution Systems Reconfiguration Based on OPF Using Benders Decomposition", IEEE Transactions on Power Delivery, vol. 24, no. 4, pp. 21662176.

LIU, Y., ZHANG, P., QIU, X. (2002) "Optimal volt/var control in distribution systems," International Journal of Electrical Power E Energy Systems, vol. 24, no. 4, pp. 271-276.

HSU, Y. (1998) "A combined artificial neural network-fuzzy dynamic programming approach to reactive power/voltage control in a distribution substation", IEEE Transactions on Power Systems, vol. 13, no. 4, pp. 1265-1271.

JUNGES, M. (2000) Lógica fuzzy em controle de tensão e potência reativa em sistemas de potência, M.Sc. Thesis, Pontifícia Universidade Católica de Minas Gerais, no. 621.31J95|T PucMinas Library, Belo Horizonte, Brazil.

EKEL, P., TERRA, L., JUNGES, M., POPOV, V. (1998) "Fuzzy technology in the design, planning and control of power systems and subsystems", Proceedings of EUFIT'98 - European Congress on Intelligent Techniques and Soft Computing, vol. 2, pp. 1126-1130, Aachen, Alemanha.

LIU, C.W., CHANG, C.S., JIANG, J.A. (2001) "Genetic algorithms as a reactive power source dispatching aid for voltage security enhancement," Proceedings of National Sci Council ROC(A), vol. 25 , no. 1 , pp. 53-62.

LIANG, R. (2003) "Fuzzy-based reactive power and voltage control in a distribution system," IEEE Transactions on Power Delivery, vol. 18, no. 2, pp. 610-618.

PEREIRA, J., SARAIVA, J.T., PONCE DE LEÃO, M.T. (1999) "Identification of operation strategies of distribution networks using a simulated annealing approach", Proceedings of IEEE Power Tech'99, Budapest, Hungary.

MIRANDA, V., FONSECA, N. (2002) "New Evolutionary Particle Swarm Algorithm (EPSO) Applied to Voltage /VAR control", Proceedings 14th PSCC - Power Systems Computation Conference, Sevilha, Espanha.

MIRANDA, V., CALISTO, P. (2002) "A fuzzy inference system to voltage/VAR control in DMS distribution management systems", Proceedings 14th PSCC - Power Systems Computation Conference, Sevilha, Espanha.

MIRANDA, V., MOREIRA, A., PEREIRA, J. (2007) "An Improved Fuzzy Inference System for Voltage/VAR Control”, IEEE Transactions on Power Systems, vol. 22, no. 4, pp. 20132020.

KENNEDY, J., EBERHART, R. (1995) "Particle swarm optimization", Proceedings of IEEE International Conference on Neural Networks, vol.4, pp.1942-1948.

BHOOMESH, R., AH KING, R.T.F., RUGHOOPUTH, H.C.S. (2003) "A modified genetic algorithm for optimal electrical distribution network reconfiguration", in CEC 'O3 - The 2003 Congress on Evolutionary Computation, vol. 2, pp. 1472-1479. 